\title{
A fuzzy inventory model with unit production cost, time depended holding cost, with-out shortages under a space constraint: a parametric geometric programming approach
}

\author{
Wasim Akram Mandal ${ }^{1}$ and Sahidul Islam ${ }^{2}$ \\ ${ }^{1}$ Beldanga Darul H.Sr.Madrasah, Beldanga, Murshidabad, India \\ ${ }^{2}$ Department of mathematics, University of kalyani, kalyani, India
}

Received: 8 February 2016, Accepted: 8 December 2016

Published online: 20 October 2017.

\begin{abstract}
In this paper, an Inventory model with unit production cost, time depended holding cost, with-out shortages is formulated and solved. We have considered a single objective structural optimization model. In most real world situation, the objective and constraint function of the decision makers are imprecise in nature. Hence the coefficients, indices, the objective function and constraint goals are imposed here in fuzzy environment. Geometric programming provides a powerful tool for solving a variety of imprecise optimization problems. Here we use nearest interval approximation method to convert a triangular fuzzy number to an interval number. In this paper, we transform this interval number to a parametric interval-valued functional form and then solve the parametric problem by geometric programming technique. Numerical example is given to illustrate the model through this Parametric Geometric-Programming method.
\end{abstract}

Keywords: Inventory model, fuzzy number, space constraint, geometric programming, interval-valued function.

\section{Introduction}

An inventory deal with decision that minimum the total average cost or maximize The total average profit. For this purpose the task is to construct a mathematical model of the real life Inventory system, such a mathematical model is based on various assumption and approximation.

In ordinary inventory model it consider all parameter like set-up cost, holding cost, interest cost a fixed. But in real life situation it will have some little fluctuations. So consideration of fuzzy variables is more realistic.

Geometric Programming (GP) method is an effective method used to solve a non-linear programming problem like structural problem. It has certain advantages over the other optimization methods. Here, the advantage is that it is usually much simpler to work with the dual than the primal one. Solving a non-linear programming problem by GP method with degree of difficulty (DD) plays essential role. (It is defined as DD = total number of terms in objective function and constraints - total number of decision variables -1$)$.

The study of inventory model where demand rates varies with time is the last decades. Geometric Programming (GP) is one of the effective methods to solve a particular type of Non linear programming problem. Geometric programming is introduced by Zener (1971) and it further developed by Duffin. Duffin R J, Peterson E L, Zener C M[6](19670) studied Geometric Programming-Theory and Application. Park and Wang studied shortages and partial backlogging of items. Friedman(1978) presented continuous time inventory model with time varying demand. Ritchie(1984) studied in inventory model with linear increasing demand. Goswami, Chaudhuri(1991) discussed an inventory model with 
shortage. Gen et. Al. (1997) considered classical inventory model with Triangular fuzzy number. Yao and Lee(1998) considered an economic production quantity model in the fuzzy sense. Sujit Kumar De, P.K.Kundu and A.Goswami(2003) presented an economic production quantity inventory model involving fuzzy demand rate. J.K.Syde and L.A.Aziz(2007) applied sign distance method to fuzzy inventory model without shortage . D.Datta and Pravin Kumar published several paper of fuzzy inventory with or without shortage. S. Islam, T.K. Roy [13](2006)presented a fuzzy EPQ model with flexibility and reliability consideration and demand depended unit Production cost under a space constraint. Kotba.M Kotb, Halla.Fergancy[8] (2011), presentedMulti-item EOQ model with both demand-depended unit cost and varying Lead time via Geometric Programming.

In this paper we first consider crisp inventory model. There after it transformed to fuzzy inventory mode and developed. First we solved the model b Fuzzy Max-Min Geometric-Programming technique and then it solved by Fuzzy parametric Geometric -Programming technique. At last it made an example and solved it by both Technique.

\section{Mathematical model}

An Inventory model is developed under the following notations and assumptions.

\subsection{Notations}

$\mathrm{I}(\mathrm{t})$ :Inventory level at any time, $\mathrm{t} \geq 0$.

D: Demand per unit, time which is constant.

T:Cycle of length.

S: Set-up cost per unit time.

$\mathrm{H}$ :Holding cost per unit item, which is time depended.

$\mathrm{P}$ :Unit demand and set-up cost dependent production cost.

q:Production quantity per batch.

$\mathrm{f}(\mathrm{D}, \mathrm{S})$ : Unit production cost per cycle.

TAC(D,S,q):Total average cost per unit time.

$\mathrm{w}_{0}$ : Space area per unit quantity.

W: Total storage space area.

\subsection{Assumptions}

(a) The inventory system involves only one item.

(b) The replenishment occur instantaneously at infinite rate.

(c) The lead time is negligible.

(d) Demand rate is constant. (e) The unit production cost is continuous function of demand and Set-up cost and take the following form

$$
P=\theta D^{-x} S^{-1}, \theta, x \in R,(>0)
$$

(f) Holding cost is time depended, as at.

\subsection{Crisp model}

The differential equation describing $I(t)$ as follows

$$
\frac{d I(t)}{d t}=-D, 0 \leq t \leq T
$$




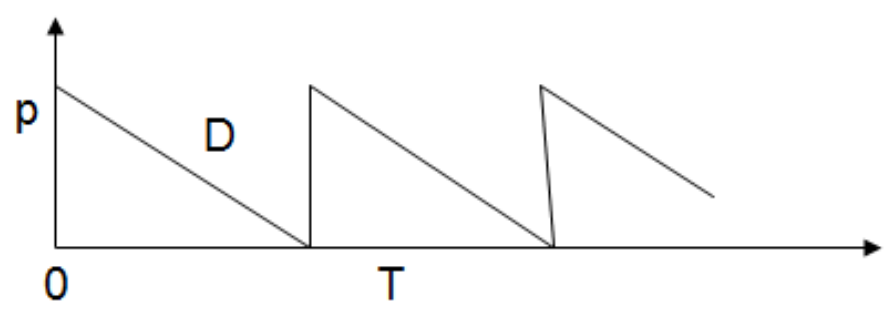

Fig. 1: Inventory Model.

with the boundary condition $I(0)=q, I(T)=0$. The solution of 1 is obtained as

$$
I(t)=q-D t
$$

Also there are

$$
T=q / D
$$

Now inventory holding cost

$$
H \int_{0}^{T} a t . I(t) d t=\frac{a H q^{3}}{6 D^{2}}
$$

Total inventory related cost per cycle $=$ set-up cost + holding cost + production cost

$$
S+\frac{a H q^{3}}{6 D^{2}}+P q
$$

So total average cost per cycle is given by

$$
T A C(D, S, q)=\frac{S D}{q}+\frac{a H q^{2}}{6 D}+\theta D^{1-x} S^{-1}
$$

and

$$
\text { storagearea }=w_{0} q .
$$

Hence the inventory model can be written as

$$
\operatorname{MinTAC}(D, S, q)=\frac{S D}{q}+\frac{a H q^{2}}{6 D}+\theta D^{1-x} S^{-1}
$$

subject to $w_{0} q \leq W, D, S, q>0$.

\subsection{Fuzzy model}

When the objective and constraint goals, coefficients and exponents become fuzzy sets and fuzzy numbers respectively, the crisp model 6 written to be a fuzzy model, as

$$
\begin{aligned}
& \tilde{\operatorname{MinTAC}}(D, S, q)=\frac{S D}{q}+\frac{\tilde{a} \tilde{H} q^{2}}{6 D}+\tilde{\theta} D^{1-x} S^{-1} \\
& \text { subject to } w_{0} q \lesssim \tilde{W}, D, S, q>0 .
\end{aligned}
$$




\section{Geometric programming (GP) problem}

Primal program: Primal Geometric Programming (PGP) problem is

$$
\text { Minimize } g_{0}(t)=\sum_{k=1}^{T_{0}} C_{0 k} \prod_{j=1}^{m} t_{j}^{\alpha_{0 k j}}
$$

subject to

$$
\sum_{k=1+T_{r-1}}^{T_{0}} C_{r k} \prod_{j=1}^{m} t_{j}^{\alpha_{r k j}} \leq 1,(r=1,2, \ldots, l), t_{j}>0,(j=1,2, \ldots, m)
$$

where $C_{0 k}(>0)\left(k=1,2, \ldots \ldots, T_{0}\right), C_{r k}(>0)$ and $\alpha_{r k j}\left(k=1,2, \ldots, 1+T_{r-1}, \ldots . ., T_{r} ; r=0,1,2, \ldots \ldots, l ; j=1,2, \ldots, m\right)$ are real numbers.

It is constrained polynomial PGP problem. The number of term each polynomial constrained functions varies and it is denoted by $\mathrm{T}_{r}$ for each $r=0,1,2, \ldots, l$. Let $T=T_{0}+T_{1}+T_{2}+\ldots T_{l}$ be the total number of terms in the primal program. The Degree of Difficulty is $(D D)=T-(m+1)$.

Dual program: Dual programming (DP) problem of 1 is:

$$
\text { Maximize }=\prod_{r=0}^{l} \prod_{k=1}^{T^{r}}\left(\frac{C_{r k}}{\delta_{r k}}\right)^{\delta_{r k}}\left(\sum_{s=1+T_{r-1}}^{T} \delta_{r s}\right)^{\delta_{r k}}
$$

subject to

$$
\begin{aligned}
& \sum_{k=1}^{T_{0}} \delta_{0 k}=1 \quad \text { (Normality condition) } \\
& \sum_{r=0}^{l} \sum_{k=1}^{T_{r}} \alpha_{r k j} \delta_{r k}=0, \quad \text { (Orthogonality conditions) } \\
& \delta_{r k}>0,\left(k=1,2, \ldots, T_{r}\right) \quad \text { (Positivity constant) }
\end{aligned}
$$

Case 1 For $\mathrm{T}_{0} \geq \mathrm{M}+1$, the dual program presents a system of linear equations for the dual variables, where the number of linear equations is either less than or equal to dual variables. More or unique solution exist for the dual vectors.

Case 2 For $\mathrm{T}_{0}<\mathrm{M}+1$, the dual program presents a system of linear equations for the dual variables, where the number of linear equations is grater than the number of dual variables. In this case generally no solution vectors exists for the dual variables. However one can get an approximate solution vector for the system using either the Latest Square(SQ) or Max-Min(MN) method.

\subsection{Solution procedure of crisp model by geometric programming $(G . P)$ technique}

Here the primal problem is

$$
\operatorname{Min} T A C(D, S, q)=\frac{S D}{q}+\frac{\tilde{a} \tilde{H} q^{2}}{6 D}+\theta D^{1-x} S^{-1}
$$

subject to $w_{0} q \leq W, D, S, q>0$. Corresponding dual form of 9 is given by

$$
\operatorname{Max}(d \omega)=\left(\frac{1}{\omega_{1}}\right) \omega_{1}\left(\frac{a H)}{6 \omega_{2}}\right)^{\omega_{2}}\left(\frac{\theta}{\omega_{3}}\right)^{\omega_{3}}\left(\frac{w_{0}}{W \omega_{01}}\right)^{\omega_{01}} \omega_{01} \omega_{01}
$$


Subject to

$$
\begin{aligned}
& \omega_{1}+\omega_{2}+\omega_{3}=1 \\
& \omega_{1}-\omega_{3}=0 \\
& \omega_{1}-\omega_{2}+(1-x) \omega_{3}=0 \\
& -\omega_{1}+2 \omega_{2}+\omega_{01}=0
\end{aligned}
$$

$\omega_{1}, \omega_{2}, \omega_{3}, \omega_{01} \geq 0$

From 10 we get $\omega_{1}=\frac{1}{4-x}, \omega_{2}=\frac{2-x}{4-x}, \omega_{3}=\frac{1}{4-x}$, and $\omega_{01}=\frac{2 x-3}{4-x}$. Putting the values in 10 we get the optimal solution of dual problem. The values of $D, S, q$ is obtained by using the primal dual relation as follows. From primal dual relation we get

$$
\begin{aligned}
& \frac{S D}{q}=\omega_{1}^{\star} \times d^{*}(\omega) \\
& \frac{a H q^{2}}{6 D}=\omega_{2}^{\star} \times d^{*}(\omega) \\
& \theta D^{1-x} S^{-1}=\omega_{3}^{\star} \times d^{*}(\omega) \\
& \frac{w_{0} q}{W}=1
\end{aligned}
$$

The optimal solution of the model through the parametric approach is given by

$$
d^{*}(\omega)=(4-x)^{\frac{1}{4-x}}\left(\frac{a H(4-x)}{(2-x) 6}\right)^{\frac{2-x}{4-x}}(\theta(4-x))^{\frac{1}{4-x}} \times\left(\frac{w_{0}(4-x)}{W(2 x-3)}\right)^{\frac{2 x-3}{4-x}}\left(\frac{2 x-3}{4-x}\right)^{\frac{2 x-3}{4-x}}
$$

and

$$
\begin{aligned}
S^{*} & =\frac{6 \omega_{1}{ }^{\star} \omega_{2}^{\star} d^{*}(\omega)^{2}}{a H} \\
D^{*} & =\frac{a H q^{2}}{6 \omega_{2}^{\star} d^{*}(\omega)} \\
q^{*} & =\frac{W}{w_{0} .}
\end{aligned}
$$

\section{Fuzzy number and its nearest interval approximation}

\subsection{Fuzzy number}

A real number $\tilde{A}$ described as fuzzy subset on the real line $R$ whose membership function $\mu_{\tilde{A}}(x)$ has the following characteristics with $-\alpha<a_{1} \leq a_{2} \leq a_{3}<\alpha$

$$
\mu_{\tilde{A}}(x)=\left\{\begin{array}{l}
\mu_{\tilde{A}}^{L}(x), \quad \text { if } \quad a_{1} \leq x \leq a_{2} \\
\mu_{\tilde{A}}^{R}(x), \quad \text { if } a_{2} \leq x \leq a_{3} \\
0, \quad \text { otherwise }
\end{array}\right.
$$

Where $\mu_{\tilde{A}}^{L}(x):\left[a_{1}, a_{2}\right] \rightarrow[0,1]$ is continuous and strictly increasing and $\mu_{\tilde{A}}^{R}(x):\left[a_{2}, a_{3}\right] \rightarrow[0,1]$ is continuous and strictly increasing.

$\alpha-$ cut of $\tilde{A}$ : The $\alpha-$ cut of $\tilde{A}$, is defined by $A_{\alpha}=\left\{x: \mu_{A}(x)=\alpha, \alpha \geq 0 A_{\alpha}\right.$ is a non-empty bounded closed interval in $X$ and it can be denoted by $A_{\alpha}=\left[A_{L}(\alpha), A_{R}(\alpha)\right]$. Where $A_{L}(\alpha)$ and $A_{R}(\alpha)$ are the lower and upper bounds of the closed interval respectively.

Figure 2 shows a fuzzy number $\tilde{A}$ with $\alpha$-cuts $\left.A_{\alpha 1}=\left[A_{L}\left(\alpha_{1}\right), A_{R}\left(\alpha_{1}\right)\right], A_{\alpha 2}=\left[A_{L}\left(\alpha_{2}\right), A_{R} \alpha_{2}\right)\right]$. It Seen that if $\alpha_{2} \geq \alpha_{1}$ then $\left.A_{L}\left(\alpha_{2}\right) \geq A_{L} \alpha_{1}\right)$ and $A_{R}\left(\alpha_{1}\right) \geq A_{R}\left(\alpha_{2}\right)$. 


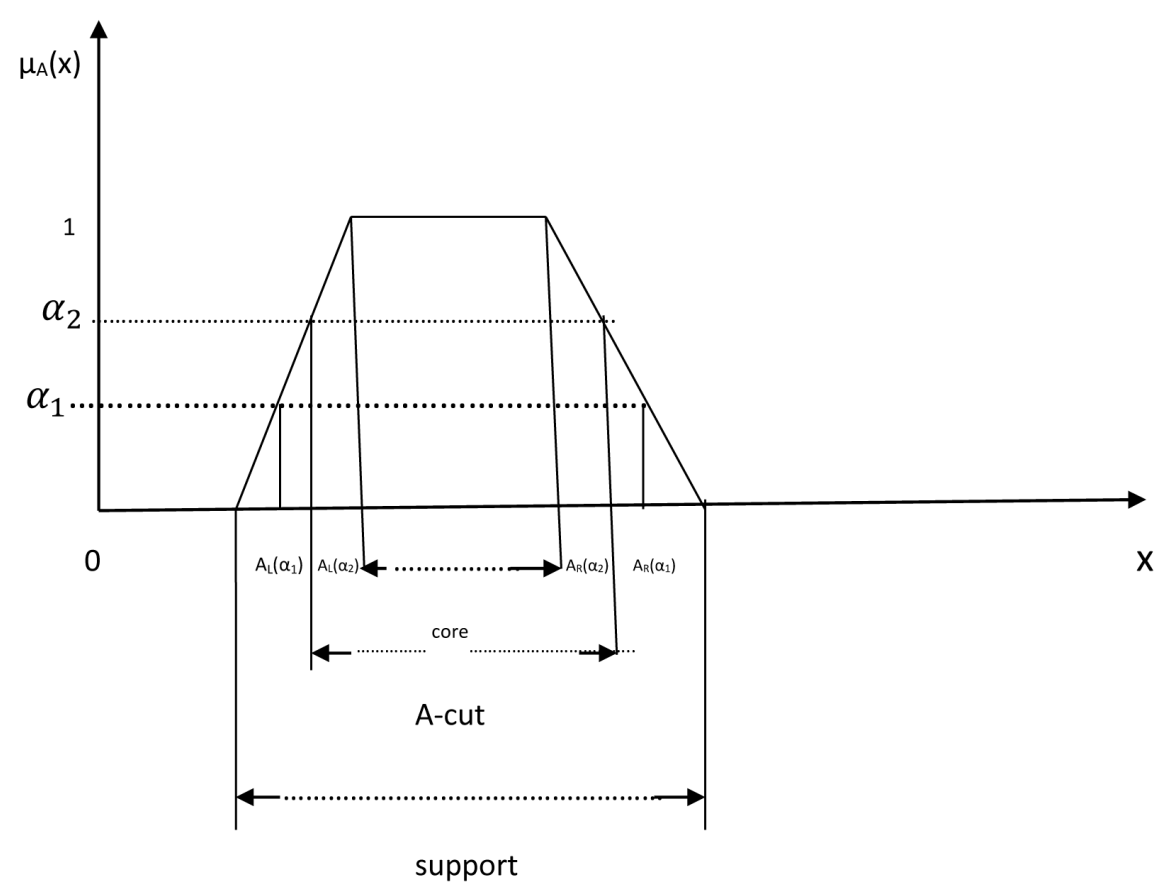

Fig. 2: Trapezoidal fuzzy number of $\tilde{A}$ with $\alpha$-cuts.

\subsection{Interval number}

An interval number $\mathrm{A}$ is defined by an ordered pair of real numbers as follows $\left.A=\left[a_{L}, a_{R}\right]=\left\{x: a_{L} \leq x \leq a_{R}\right\}, x \in R\right\}$ where and $a_{L}$ are the left and $a_{R}$ right bounds of interval A, respectively. The interval A, is also defined by center $\left(a_{c}\right)$ and half-width $\left(a_{w}\right)$ as follows.

$A=\left(a_{c}, a_{W}\right)=\left\{x: a_{c}-a_{w} \leq x \leq a_{c}+a_{w}, x \in R\right\}$ where $a_{c}=\frac{a_{R}+a_{L}}{2}$ is the center and $a_{w}=\frac{a_{R}-a_{L}}{2}$ is the half-width of A.

\subsection{Nearest interval approximation}

Here we want to approximate a fuzzy number by a crisp model. Suppose $\tilde{A}$ and $\tilde{B}$ are two fuzzy numbers with $\alpha$-cuts are $\left[A_{L}(\alpha), A_{R}(\alpha)\right]$ and $\left[B_{L}(\alpha), B_{R}(\alpha)\right]$ respectively. Then the distance between $\tilde{A}$ and $\tilde{B}$ is

$$
d(\tilde{A}, \tilde{B})=\sqrt{\int_{0}^{1}\left(A_{L}(\alpha)-B_{L}(\alpha)\right)^{2}+\int_{0}^{1}\left(A_{R}(\alpha)-B_{R}(\alpha)\right)^{2} d \alpha}
$$

with respect to $C_{L}$ and $C_{R}$. In order to minimize $d\left(\tilde{A}, C_{D}(\tilde{A})\right)$, it is sufficient to minimize the function $D\left(C_{L}, C_{R}\right)=\left(d^{2}\left(\tilde{A}, \tilde{C}_{D}(\tilde{A})\right)\right)$. The first partial derivatives are

$$
\frac{\partial}{\partial C_{L}} \mathrm{D}\left(C_{L}, C_{R}\right)=-2 \int_{0}^{1} \mathrm{~A}_{\mathrm{L}}(\alpha) \mathrm{d} \alpha+2 C_{L}
$$

And

$$
\frac{\partial}{\partial C_{R}} \mathrm{D}\left(C_{L}, C_{R}\right)=-2 \int_{0}^{1} \mathrm{~A}_{\mathrm{R}}(\alpha) \mathrm{d} \alpha+2 C_{R}
$$

Solving $\frac{\partial}{\partial C_{L}} \mathrm{D}\left(C_{L}, C_{R}\right)=0$ and $\frac{\partial}{\partial C_{R}} \mathrm{D}\left(C_{L}, C_{R}\right)=0$, we get $C_{L}=\int_{0}^{1} A_{L}(\alpha) d \alpha$ and $C_{R} \int_{0}^{1} A_{R}(\alpha) d \alpha$. Again since

$$
\frac{\partial^{2}}{\partial C_{L}^{2}}\left(D\left(C_{L}{ }^{*}, C_{R}{ }^{*}\right)\right)=2>0, \frac{\partial^{2}}{\partial C_{R}{ }^{2}}\left(D\left(C_{L}^{*}, C_{R}{ }^{*}\right)\right)=2>0
$$


and

$$
H\left(C_{L}^{*}, C_{R}^{*}\right)=\frac{\partial^{2}}{\partial C_{L}^{2}}\left(D\left(C_{L}^{*}, C_{R}^{*}\right)\right) \cdot \frac{\partial^{2}}{\partial C_{R}^{2}}\left(D\left(C_{L}^{*}, C_{R}^{*}\right)\right)-\left(\frac{\partial^{2}}{\partial C_{L}^{*} C_{L}^{*}}\left(D\left(C_{L}^{*}, C_{R}^{*}\right)\right)\right)^{2}=4>0
$$

So $D\left(C_{L}^{*}, C_{R}^{*}\right)$ i.e. $d\left(\tilde{A}, C_{D}(\tilde{A})\right)$ is global minimum. Therefore, the interval $C_{d}(\tilde{A})=\left[\int_{0}^{1} A_{L}(\alpha) d \alpha, \int_{0}^{1} A_{R}(\alpha) d \alpha\right]$ is the nearest interval approximation of fuzzy number $\tilde{A}$ with respect to the metric $d$. Let $\tilde{A}=\left(a_{1}, a_{2}, a_{3}\right)$ be a triangular fuzzy number. The $\alpha$-cut interval of $\tilde{A}$ is defined as $A_{\alpha}=\left[A_{L}(\alpha), A_{R}(\alpha)\right]$ where $A_{L}(\alpha)=a_{1}+\alpha\left(a_{2}-a_{1}\right)$ and $A_{R}(\alpha)=a_{3}-\alpha\left(a_{3}-a_{2}\right)$. By nearest interval approximation method the lower limit of the interval is

$$
C_{L}=\int_{0}^{1} A_{L}(\alpha) d \alpha=\int_{0}^{1}\left[a_{1}+\alpha\left(a_{2}-a_{1}\right)\right] d \alpha=\frac{a_{1}+a_{2}}{2}
$$

and the upper limit of the interval is

$$
C_{R}=\int_{0}^{1} A_{R}(\alpha) d \alpha=\int_{0}^{1}\left[a_{3}-\alpha\left(a_{3}-a_{2}\right)\right] d \alpha=\frac{a_{3}+a_{2}}{2} .
$$

Therefore, the interval number corresponding $\tilde{A}$ is $\left[\frac{a_{1}+a_{2}}{2}, \frac{a_{3}+a_{2}}{2}\right]=[m, n]$ In the centre and half -width form the interval number of $\tilde{A}$ is defined as $\left\langle\frac{1}{4}\left(a_{1}+2 a_{2}+a_{3}\right), \frac{1}{4}\left(a_{3}-a_{1}\right)\right\rangle$.

\subsection{Parametric Interval-valued function}

Let $[m, n]$ be an interval, where $m>0, n>0$. From analytical geometry point of view, any real number can be represented on a line. Similarly; we can express an interval by a function. The parametric interval-valued function for the interval $[m, n]$ can be taken as $g(s)=m^{1-s} n^{s}$ for $s \in[0,1]$, which is strictly monotone, continuous function and its inverse exits. Let $\psi$ be the inverse of $g(s)$, then

$$
s=\frac{\log \psi-\log m}{\log n-\log m} .
$$

\subsection{Geometric Programming with fuzzy coefficient}

When all coefficients of Eq. (6) are triangular fuzzy number, then the geometric programming problem is of the form

$$
\min \tilde{g}_{0}(x)
$$

subject to $\tilde{g}_{i}(x) \lesssim 1(1 \leq i \leq n), x>0$. Its objective function is

$$
\tilde{g}_{0}(x)=\sum_{k=1}^{T_{i}} \tilde{c}_{0 k} \prod_{j=1}^{m} x_{j}^{\alpha_{0 k j}}
$$

and constraints of the form

$$
\tilde{g}_{i}(x)=\sum_{k=1}^{T_{i}} \tilde{c}_{i k} \prod_{j=1}^{m} x_{j}^{\alpha_{i k j}} \quad(1 \leq i \leq n)
$$

are all polynomials of $x$ in which coefficients $\tilde{c}_{0 k}$ and indexes $\tilde{c}_{i k}$ are fuzzy numbers. Where $\tilde{c}_{0 k}=\left(c_{0 k}^{1}, c_{0 k}^{2}, c_{0 k}^{3}\right)$ and $\tilde{c}_{i k}=\left(c_{i k}^{1}, c_{i k}^{2}, c_{i k}^{3}\right)$. Using nearest interval approximation method, we transform all triangular fuzzy number into interval number i.e. $\left[c_{0 k}^{L}, c_{0 k}^{U}\right]$ and $\left[c_{i k}^{L}, c_{i k}^{U}\right]$. The geometric programming problem with imprecise parameters is of the following form

$$
\min \hat{g}_{0}(x)
$$

subject to $\hat{g}_{i}(x) \lesssim 1(1 \leq i \leq n), x>0$.

Its objective function is

$$
\hat{g}_{0}(x)=\sum_{k=1}^{T_{i}} \hat{c}_{0 k} \prod_{j=1}^{m} x_{j}^{\alpha_{0 k j}}
$$


and constraints of the form

$$
\hat{g}_{i}(x)=\sum_{k=1}^{T_{i}} \hat{c}_{i k} \prod_{j=1}^{m} x_{j}^{\alpha_{i k j}}(0 \leq i \leq n)
$$

where $\hat{c}_{i k}$ and $\hat{b}_{i}$ denote the interval counterparts i.e. $\hat{c}_{i k} \in\left[c_{0 k}^{L}, c_{0 k}^{U}\right]$ and $\hat{c}_{i k} \in\left[c_{i k}^{L}, c_{i k}^{U}\right] . c_{0 k}^{L}>0, c_{0 k}^{L}>0$ for all $i$ and $k$. Using parametric interval-valued functional form, the problem reduces to

$$
\min g_{0}(x, s)=\sum_{k=1}^{T_{0}}\left(c_{0 k}^{L}\right)^{1-s}\left(c_{0 k}^{U}\right)^{S} \prod_{j=1}^{m} x_{j}^{\alpha_{0 k j}}
$$

Subject to

$$
g_{i}(x, s)=\sum_{k=1}^{T_{i}}\left(c_{i k}^{L}\right)^{1-s}\left(c_{i k}^{U}\right)^{S} \prod_{j=1}^{m} x_{j}^{\alpha_{i k j}} \leq 1, x_{i}>0 \quad \text { for } \quad i=1,2, \ldots \ldots . n, j=1,2, \ldots \ldots \ldots .
$$

This is a parametric geometric programming problem. We get different solutions of this problem for different value of the parameter $s$.

\subsection{Solution procedure of fuzzy model by Geometric Programming (G.P) technique}

When $\tilde{a}=\left(a_{1}, a_{2}, a_{3}\right), \tilde{H}=\left(H_{1}, H_{2}, H_{3}\right), \tilde{\theta}=\left(\theta_{1}, \theta_{2}, \theta_{3}\right)$ and $\tilde{W}=\left(W_{1}, W_{2}, W_{3}\right)$ are triangular fuzzy number .then the fuzzy model is

$$
\text { (Min) } T A C(D, S, q)=S D / q+\left(\tilde{a} \tilde{H} q^{2}\right) / 6 D+\tilde{\theta} D^{(1-x)} S^{-1}
$$

subject to

$$
w_{0} q \lesssim \tilde{W}, D, S, q>0 .
$$

Using nearest interval approximation method, the interval number corresponding triangular number $\tilde{a}=\left(a_{1}, a_{2}, a_{3}\right)$ is

$$
\left[\left(a_{1}+a_{2}\right) / 2,\left(a_{3}+a_{2}\right) / 2\right]=\left[a_{L}, a_{U}\right] .
$$

Similarly interval number corresponding $\tilde{H} \tilde{\theta}$ and $\tilde{W}$ are

$$
\left[\left(H_{1}+H_{2}\right) / 2,\left(H_{3}+H_{2}\right) / 2\right]=\left[H_{L}, H_{U}\right],\left[\left(\theta_{1}+\theta_{2}\right) / 2,\left(\theta_{3}+\theta_{2}\right) / 2\right]=\left[\theta_{L}, \theta_{U}\right]
$$

and

$$
\left[\left(W_{1}+W_{2}\right) / 2,\left(W_{3}+W_{2}\right) / 2\right]=\left[W_{L}, W_{U}\right]
$$

respectively. The problem (11) reduces to

$$
\operatorname{Min} \operatorname{TAC}(D, S, q)=S D / q+\left(\left[a_{L}, a_{U}\right]\left[H_{L}, H_{U}\right] q^{2}\right) / 6 D+\left[\theta_{L}, \theta_{U}\right] D^{1-x)} S^{-1}
$$

subject to

which is equivalent to

$$
w_{0} q \leq\left[W_{L}, W_{U}\right], D, S, q>0 .
$$

$$
\operatorname{Min} \operatorname{TAC}(D, S, q)=S D / q+\left(\hat{a} \hat{H} q^{2}\right) / 6 D+\hat{\theta} D^{(1-x)} S^{(-1)}
$$

subject to

$$
w_{-} 0 q \lesssim \hat{W}, D, S, q>0 .
$$

where $\hat{a} \in\left[a_{L}, a_{U}\right], \hat{H} \in\left[H_{-} L, H_{U}\right], \hat{\theta} \in\left[\theta_{L}, \theta_{U}\right]$ and $\hat{W} \in\left[W_{L}, W_{U}\right]$.

According to section 4, the fuzzy model (13) reduces to a parametric programming by replacing $\hat{a}=a_{L}^{1-s} a_{U}^{s}, \hat{H}=H_{L}^{1-s} H_{U}^{s}, \hat{\theta}=\theta_{L}^{1-s} \theta_{U}^{s}, \hat{W}=W_{L}^{1-s} W_{U}^{s}$ and where $s \in[0,1]$.

The model takes the reduces form as follows

$$
\operatorname{Min} \operatorname{TAC}(D, S, q)=S D / q+\left(\left(a_{L}^{(1-s)} a_{U}^{s}\right)\left(H_{L}^{(1-s)} H_{U}^{s}\right) q^{2}\right) / 6 D+\left(\theta_{L}^{(1-s)} \theta_{U}^{s}\right) D^{(1-s)} S^{(-1)}
$$


subject to

$$
w_{0} q \lesssim\left(W_{L}^{(1-s)} W_{U}^{s}\right), D, S, q>0
$$

Corresponding dual form of 9 is given by

$$
\operatorname{Maxd}(\omega)=\left(\frac{1}{\omega_{1}}\right)^{\omega_{1}}\left(\frac{\left(a_{L}^{1-s} a_{U}^{s}\right)\left(H_{L}{ }^{1-s} H_{U}\right)^{s}}{6 \omega_{2}}\right)^{\omega_{2}}\left(\frac{\left(\theta_{L}^{1-s} \theta_{U}\right)^{\prime}}{\omega_{3}}\right)^{\omega_{3}}\left(\frac{w_{0}}{\left(W_{L}^{1-s} W_{U}^{s}\right) \omega_{01}}\right)^{\omega_{01}} \omega_{01} \omega_{01}
$$

subject to

$$
\begin{aligned}
& \omega_{1}+\omega_{2}+\omega_{3}=1 \\
& \omega_{1}-\omega_{3}=0 \\
& \omega_{1}-\omega_{2}+(1-x) \omega_{3}=0 \\
& -\omega_{1}+2 \omega_{2}+\omega_{01}=0 \\
& \omega_{1}, \omega_{2}, \omega_{3}, \omega_{01} \geq 0 .
\end{aligned}
$$

From 13 we get $\omega_{1}=\frac{1}{4-x}, \omega_{2}=\frac{2-x}{4-x}, \omega_{3}=\frac{1}{4-x}$, and $\omega_{01}=\frac{2 x-3}{4-x}$. Putting the values in 13 we get the optimal solution of dual problem. The values of $D, S, q$ is obtained by using the primal dual relation as follows. From primal dual relation we get

$$
\begin{aligned}
& S D / q=\omega_{1}^{*} \times d^{*}(\omega) \\
& \left(a_{L}^{(1-s)} a_{U}^{s}\right)\left(H_{L}^{(1-s)} H_{U} s\right) q^{2} / 6 D=\omega_{2}^{*} \times d^{*}(\omega) \\
& \left(\theta_{L}^{(1-s)} \theta_{U}^{s}\right) D^{(1-x)} S^{(-1)}=\omega_{3}^{*} \times d^{*}(\omega) \\
& \left(w_{0} q\right) /\left(\left(W_{L}^{(1-s)} W_{U} s\right)\right)=1 .
\end{aligned}
$$

The optimal solution of the model through the parametric approach is given by

$$
d^{*}(\omega)=(4-x)^{\frac{1}{4-x}}\left(\frac{\left(a_{L}^{1-s} a_{U}^{s}\right)\left(H_{L}^{1-s} H_{U}^{s}\right)(4-x)}{(2-x) 6}\right)^{\frac{2-x}{4-x}}\left(\left(\theta_{L}^{1-s} \theta_{U}^{s}\right)(4-x)\right)^{\frac{1}{4-x}} \times\left(\frac{w_{0}(4-x)}{\left(W_{L}^{1-s} W_{U}\right)(2 x-3)}\right)^{\frac{2 x-3}{4-x}}\left(\frac{2 x-3}{4-x}\right)^{\frac{2 x-3}{4-x}}
$$

and

$$
\begin{aligned}
S^{*} & =\frac{6 \omega_{1}^{\star} \omega_{2}^{\star} d^{*}(\omega)^{2}}{\left(a_{L}{ }^{1-s} a_{U}{ }^{s}\right)\left(H_{L}{ }^{1-s} H_{U}{ }^{s}\right)} \\
D^{*} & =\frac{\left(a_{L}{ }^{1-s} a_{U}{ }^{s}\right)\left(H_{L}{ }^{1-s} H_{U}{ }^{s}\right) q^{2}}{6 \omega_{2}^{\star} d^{*}(\omega)} \\
q^{*} & =\frac{\left(W_{L}{ }^{1-s} W_{U}{ }^{s}\right)}{w_{0}}
\end{aligned}
$$

\section{Numerical example and solution}

A manufacturing company produces a machine. It is given that the inventory carrying cost of the machine is $\$ 15$ per unit per year. The production cost of the machine varies inversely with the demand and set-up cost. From the past experience, the production cost of the machine is $120 D^{-3} S^{-1}$ where $\mathrm{D}$ is the demand rate and $\mathrm{S}$ is set-up cost. Storage space area per unit time $\left(w_{0}\right)$ and total storage space area (W) are 100 sq. ft. and 2000 sq. ft. respectively. Determine the demand rate (D), set-up cost (S), production quantity (q), and optimum total average cost (TAC) of the production system.

Then the input value of the model 6 is

Table 1

\begin{tabular}{|l|l|l|l|l|l|}
\hline $\mathrm{a}$ & $\mathrm{H}$ & $\mathrm{X}$ & $\theta$ & $w_{0}$ & $\mathrm{~W}$ \\
\hline 7 & 15 & 1.75 & 120 & 100 & 2000 \\
\hline
\end{tabular}


Then the model is of the form

$$
\begin{aligned}
& \text { Min } \operatorname{TAC}(D, S, q)=\frac{S D}{q}+\frac{105 q^{2}}{6 D}+120 D^{-0.75} S^{-1} \\
& \text { subject to } 100 q \leq 2000, D, S, q>0 .
\end{aligned}
$$

Table 2: Optimal solution of 6 for crisp model.

\begin{tabular}{|l|l|l|l|l|}
\hline $\begin{array}{l}\text { Crisp } \\
\text { model }\end{array}$ & $\mathrm{S}^{*}$ & $\mathrm{D}^{*}$ & $\mathrm{q}^{*}$ & $\mathrm{TAC}^{*}\left(\mathrm{~S}^{*}, \mathrm{D}^{*}, \mathrm{q}^{*}\right) \$$ \\
\hline G.P & 0.684 & 4048 & 20 & 140.517 \\
\hline N.L.P & 0.685 & 4047 & 20 & 140.685 \\
\hline
\end{tabular}

When the input data of inventory model is taken as triangular fuzzy number i.e.

$$
\begin{aligned}
& \left.a=[6,8], \Rightarrow a=(6)^{(} 1-s\right)(8)^{s} \in[6,8] \\
& H=[14,16], \Rightarrow \hat{H}=(14)^{(1-s)}(16)^{s} \in[14,16] \\
& \left.\theta=[118,122], \hat{\theta}=(118)^{(} 1-s\right)(122)^{s} \in[118,122] \\
& \left.W=[1900,2100], \Rightarrow W=(1900)^{(} 1-s\right)(2100)^{s} \in[1900,2100] \text {, where } s \in[0,1] \text {. }
\end{aligned}
$$

Table 3: Optimal Solution of Fuzzy Inventory Model.

\begin{tabular}{|ccccc|}
\hline$S$ & $S^{*}$ & $D^{*}$ & $q^{*}$ & $T C^{*}\left(S^{*}, D^{*}, q^{*}\right) \$$ \\
\hline 0.0 & 0.820 & 2983.86 & 21.00 & 119.801 \\
0.1 & 0.786 & 3175.16 & 20.79 & 123.060 \\
0.2 & 0.753 & 3378.71 & 20.58 & 126.396 \\
0.3 & 0.722 & 3595.32 & 20.38 & 129.924 \\
0.4 & 0.693 & 3825.81 & 20.18 & 133.735 \\
0.5 & 0.664 & 4071.08 & 19.97 & 137.533 \\
0.6 & 0.637 & 4332.07 & 19.78 & 141.519 \\
0.7 & 0.610 & 4609.80 & 19.58 & 145.473 \\
0.8 & 0.585 & 4905.33 & 19.38 & 19.38 \\
0.9 & 0.561 & 5219.81 & 19.19 & 154.196 \\
1.0 & 0.538 & 5554.45 & 19.00 & 158.767 \\
\hline
\end{tabular}

Here we have given a rough graph, which shown how change the value of $T A C *(S *, D *, q *)$ for different values of $s$.

\section{Sensitivity analysis}

Effect, for increment of parameter $s$.

1. For increasing value of " $s$ ", set-up $S^{*}$ cost decreasing.

2. For increasing value of "s", demand $\mathrm{D}^{*}$ rate increasing.

3. For increasing value of "s, Production quantity $q^{*}$ decreasing.

4. For increasing value of " $s "$, Total average cost $T A C^{*}\left(S^{*}, D^{*}, q^{*}\right)$ increasing.

\section{Conclusion}

In this paper, we have proposed a real life inventory problem in a fuzzy environment and presented solution along with sensitivity analysis approach. The inventory model developed with unit production cost, time depended holding cost, with-out shortages. This 


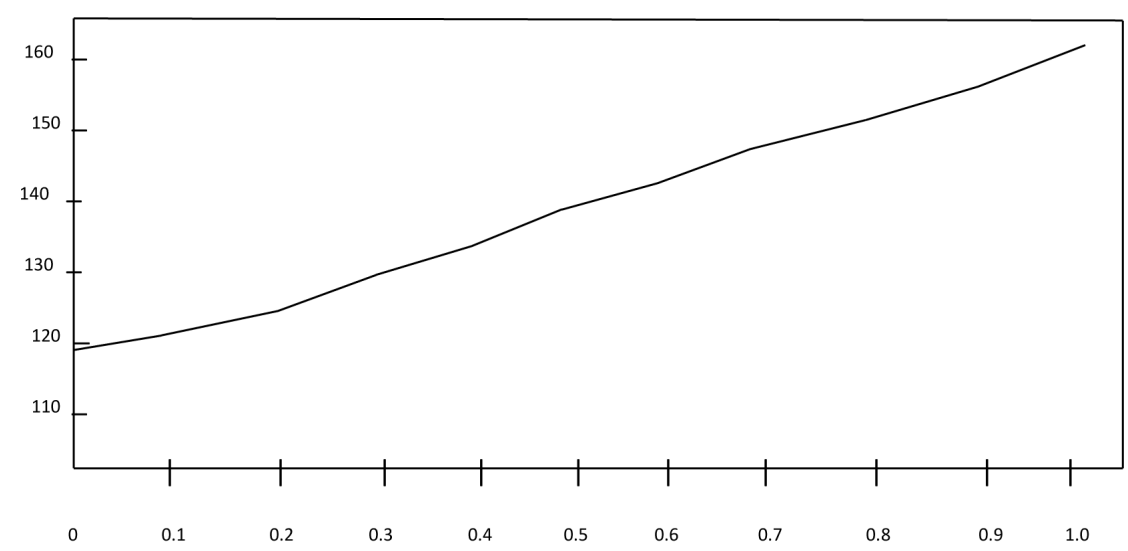

Fig. 3: Change of the value of objective function for change of $s$ by Fuzzy Geometric Programming Technique.

model has been developed for single item.

In this paper, we first create a crisp model then it transformed to fuzzy model and solved by parametric Geometric-Programming technique.Here decision maker may obtain the optimal results according to his expectation .In fuzzy we have considered triangular fuzzy number(T.F.N) In future, the other type of membership functions such as piecewise linear hyperbolic, L-R fuzzy number, Trapezoidal Fuzzy Number (TrFN), Parabolic flat Fuzzy Number (PfFN), Parabolic Fuzzy Number (pFN), pentagonal fuzzy number etc can be considered to construct the membership function and then model can be easily solved.

\section{Competing interests}

The authors declare that they have no competing interests.

\section{Authors' contributions}

All authors have contributed to all parts of the article. All authors read and approved the final manuscript.

\section{References}

[1] Bellman, R.E, and Zadeh (1970), Decision making in a fuzzy environment, Management Science 17, B141-B164.

[2] Carlsson, C. and P. Korhonen (1986), A parametric approach to fuzzy linear programming, Fuzzy sets and systems, 17-30.

[3] Clark, A.J, (1992), An informal survey of multy-echelon inventory theory , naval research logistics Quarterly 19, 621-650.

[4] D.Dutta and Pavan Kumar (2012), Fuzzy inventory without shortages using trapezoidal fuzzy number with sensitivity analysis IOSR Journal of mathematics, Vol. 4(3), 32-37.

[5] Dutta, D.J.R. Rao, and R.N Tiwary (1993), Effect of tolerance in fuzzy linear fractional programming, fuzzy sets and systems 55, 133-142.

[6] Duffin,R.J., Peterson,E.L. \& Zener,C.M. (1967).Geometric programming- theory and applications. Wiley, New York.

[7] Hamacher, H.Leberling and H.J.Zimmermann (1978), Sensitivity Analysis in fuzzy linear Programming Fuzzy sets and systems 1, 269-281.

[8] Hadley, G. and T.M. White (1963),Analysis of inventory system, Prentice-Hall, Englewood Cliffs, NJ.

[9] Kotb A.M Kotb, Hala A.Fergancy (2011), Multi-item EOQ model with both demand-depended unit cost and varying Leadtime via Geometric Programming, Applied mathematics, 2011, 2, 551-555.

[10] Khun, H.W and A.W. Tucker (1951), Non-linear programming, proceeding second Berkeley symposium Mathematical Statistic and probability (ed) Nyman ,J.University of California press 481-492. 
[11] Li,H.X. and Yen, V.C. (1995), Fuzzy Sets and Fuzzy decision making, CRC press, London.

[12] M.K.Maity (2008), Fuzzy inventory model with two ware house under possibility measure in fuzzy goal, Euro.J.Oper. Res 188,746774.

[13] Raymond, F.E (1931), Quantity and Economic in manufacturing, McGraw-Hill, New York.

[14] S. Islam, T.K. Roy (2006), A fuzzy EPQ model with flexibility and reliability consideration and demand depended unit Production cost under a space constraint: A fuzzy geometric programming approach, Applied Mathematics and Computation vol. 176(2), 531-544.

[15] S. Islam, T.K. Roy (2010), Multi-Objective Geometric-Programming Problem and its Application. Yugoslav Journal of Operations Research,20,213-227.

[16] S.T.Liu (2006), Posynomial Geometric-Programming with interval exponents and co-efficients, Europian Journal of Operations Research,168(2006), no.2, 345-353.

[17] T.K. Roy and M. Maity (1995), A fuzzy inventory model with constraints, Opsearch, 32(4) (1995) 287-298.

[18] Y.Liang, F.Zhou (2011), A two warehouse inventory model for deteriorating items under conditionally permissible delay in Payment, Appl. Math. Model.35, 2221-2231.

[19] Zadeh, L.A (1965), Fuzzy sets, Information and Control, 8, 338-353.

[20] Zimmermann, H.J.(1985),Application of fuzzy set theory to mathematical programming, Information Science, 36, $29-58$.

[21] Zimmermann, H.J.(1992), "Methods and applications of Fuzzy Mathematical programming”, in An introduction to Fuzzy Logic Application in Intelligent Systems (R.R. Yager and L.A.Zadeh, eds), pp.97- 120, Kluwer publishers, Boston. 